\title{
Robust MMSE Precoding Strategy for Multiuser MIMO Relay Systems with Switched Relaying and Side Information
}

\author{
Yunlong Cai ${ }^{\# 1}$, Rodrigo C. de Lamare ${ }^{* 2}$, Lie-Liang Yang \&3 and Minjian Zhao ${ }^{* 4}$ \\ \# Department of Information Science and Electronic Engineering, Zhejiang University, Hangzhou 310027, China \\ * Department of Electronics, University of York, York, UK, YO10 5DD \\ \& School of Electronics and Computer Science, University of Southampton, SO17 1BJ Southampton, UK \\ ${ }^{1}$ ylcai@zju.edu.cn, ${ }^{2}$ rcdl500@ohm.york.ac.uk, ${ }^{3}$ lly@ecs.soton.ac.uk ${ }^{4}$ mjzhao@zju.edu.cn
}

\begin{abstract}
In this work, we propose a minimum mean squared error (MMSE) robust base station (BS) precoding strategy based on switched relaying (SR) processing and limited transmission of side information for interference suppression in the downlink of multiuser multiple-input multiple-output (MIMO) relay systems. The BS and the MIMO relay station (RS) are both equipped with a codebook of interleaving matrices. For a given channel state information (CSI) the selection function at the BS chooses the optimum interleaving matrix from the codebook based on two optimization criteria to design the robust precoder. Prior to the payload transmission the BS sends the index corresponding to the selected interleaving matrix to the RS, where the best interleaving matrix is selected to build the optimum relay processing matrix. The entries of the codebook are randomly generated unitary matrices. Simulation results show that the performance of the proposed techniques is significantly better than prior art in the case of imperfect CSI.
\end{abstract}

Index Terms- Robust precoding, switched relaying, limited feedforward, multiuser MIMO relay.

\section{INTRODUCTION}

Employing MIMO relays in multiuser cellular systems has become a hot topic of current research, since it is able to further increase the link quality, reliability, and data rate of the system. Many works have been reported for multiuser MIMO relay systems [1]-[7]. In particular, in the presence of imperfect CSI, two robust linear precoding schemes at the RS based on zero forcing (ZF) and MMSE criteria were proposed for downlink multiuser MIMO relay systems in [3]. However, the authors did not consider the source precoding in this work. An optimization strategy for the joint transmit diversity and relay selection in cooperative MIMO systems has been reported in [4]. In [5], Xu et al. proposed singular value decomposition (SVD) based joint BS and RS precoding algorithms, where the BS precoder is designed based on the SVD of the first time slot channel, and the ZF RS precoder is obtained based on the second time slot channel. By using the same theory and taking the channel estimated errors into account, the authors investigated SVD-based robust precoding schemes in [6], [7]. The precoders are designed in a decentralized way at the BS and RS, respectively. However, they are not designed based on a unified cost function to optimize the overall system performance. The SVD-based algorithms may lead to a suboptimum solution. Moreover, the iterative algorithms [8], [9] designed based on optimizing the system performance for BS and RS precoders may not be suitable for practical implementation in multiuser MIMO

${ }^{1}$ This work was supported by the Fundamental Research Funds for the Central Universities, the NSF of China under Grant 61101103 and the Scientific Research Project of Zhejiang Provincial Education Department. relay systems. Since they are centralized algorithms which have to be implemented at the BS or RS, the required RS or BS precoding information has to be sent by the control signalling channels. In particular, when the channel varies fast, the transmission efficiency becomes very low and the CSI becomes outdated. Thus, more sophisticated and efficient relaying algorithms are needed.

Prior work on switched interleaving techniques with limited feedback has been proposed in the context of code-division multiple access (CDMA) systems [10], [11]. In this work, we propose an MMSE robust BS precoding strategy based on SR processing and limited side information for downlink multiuser MIMO relay systems in the presence of imperfect CSI. The $\mathrm{BS}$ and MIMO RS are both equipped with a codebook of interleaving matrices. For a given CSI, the selection function at the BS chooses the optimum interleaving matrix from the codebook based on the minimum sum squared Euclidean distance between the true symbol value and the estimated soft decision or maximum sum rate to design the robust BS precoder. The BS feeds forward the index corresponding to the selected interleaving matrix to the RS before the payload transmission, where the best interleaving matrix is selected to build the optimum relaying processing matrix. The entries of the codebook are randomly generated unitary matrices. We devise an expression for the transmission efficiency of the proposed SR-based precoding scheme.

\section{Proposed System Model}

Consider the downlink of a multiuser MIMO cellular system consisting of one BS, one fixed RS, and $K$ mobile stations (MSs), as shown in Fig. 1. We equip the BS and the RS with a codebook of interleaving matrices. During each transmission block, the BS first chooses the optimum interleaving matrix from the codebook as the relay processing matrix based on a selection criterion and the estimated CSIs. Then, the BS determines the optimum BS precoder based on the selected interleaving matrix and sends the index of the selected entry in the codebook to the RS through a limited feedforward channel before data transmission. The selection process takes place for each transmission block. Note that we restrict our study to a simplified model, where the direct link between the BS and the MSs is neglected. The $K$ MSs are served by the BS simultaneously through a single RS. It is assumed that the BS and RS are equipped with $N_{t}$ and $N_{r}$ antennas, respectively, and each MS is equipped with a single antenna, where $K \leq \min \left\{N_{t}, N_{r}\right\}$. We consider the half time-division duplex nonregenerative relaying [12]. 


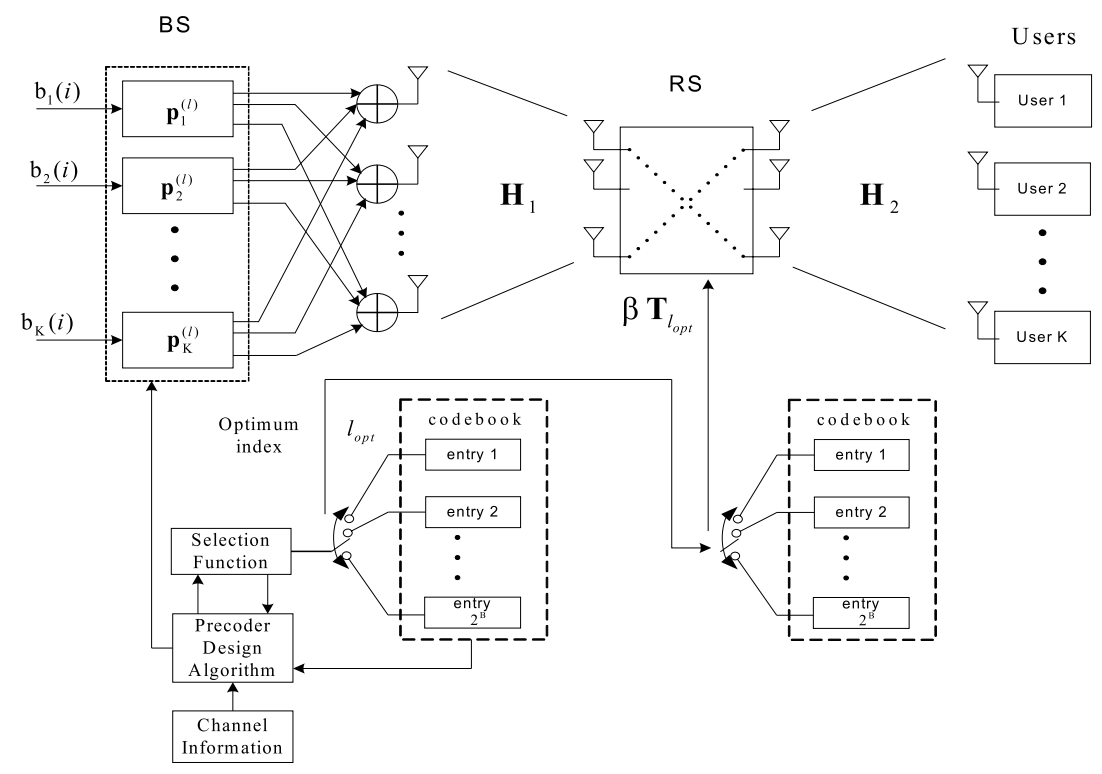

Fig. 1. Proposed linear precoding using switched relaying processing for multiuser MIMO relay systems

In the first phase, the $K$ symbols are preprocessed at the BS by the $l$-th precoder designed based on an interleaving matrix $\mathbf{T}_{l}$, where $\mathbf{T}_{l} \in \mathcal{T}=\left\{\mathbf{T}_{1}, \mathbf{T}_{2}, \ldots, \mathbf{T}_{2^{B}}\right\}$ is chosen according to the criteria that will be detailed later, where $\forall l \in\left\{1, \ldots, 2^{B}\right\}, B$ denotes the number of quantization bits which are sent to the RS before the transmission, and $2^{B}$ is the codebook size. The received vector at the RS is given by

$$
\mathbf{r}_{R}^{(l)}=\mathbf{H}_{1} \mathbf{P}_{l} \mathbf{b}+\mathbf{n}_{1}
$$

where $\mathbf{b}=\left[b_{1}, \ldots, b_{K}\right]^{T}$ is the statistically and independently generated $K \times 1$ transmit symbol vector, $E\left[\left|b_{k}\right|^{2}\right]=1$, $k \in\{1, \ldots, K\}, E[$.$] denotes the expectation operator, [.] ]^{T}$ denotes transpose, $\mathbf{P}_{l}=\left[\mathbf{p}_{1}^{(l)}, \ldots, \mathbf{p}_{K}^{(l)}\right]$ denotes the $l$-th $N_{t} \times K$ precoding matrix at the $\mathrm{BS}$, with $\mathbf{p}_{k}^{(l)}$ denoting the $l$-th $N_{t} \times 1$ precoding vector applied to the transmit symbol for user $k$. The matrix $\mathbf{H}_{1}$ is the $N_{r} \times N_{t}$ channel matrix whose elements are independent and identically distributed (i.i.d.) complex Gaussian variables with zero mean and unit variance, and $\mathbf{n}_{1}$ is the $N_{r} \times 1$ additive complex Gaussian noise with $E\left[\mathbf{n}_{1} \mathbf{n}_{1}^{H}\right]=\sigma_{1}^{2} \mathbf{I}$, where $\sigma_{1}^{2}$ denotes the first phase noise variance, $\mathbf{I}$ denotes an identity matrix of appropriate dimension, and [.] ${ }^{H}$ denotes Hermitian transpose.

In the second phase, the vector $\mathbf{r}_{R}^{(l)}$ is operated by the $l$ th $N_{r} \times N_{r}$ relay processing matrix $\mathbf{T}_{l}$, the forwarded signal vector from the RS is given by

$$
\mathbf{x}_{R}^{(l)}=\beta \mathbf{T}_{l} \mathbf{r}_{R}^{(l)},
$$

where the parameter $\beta$ is used to scale the transmit power of the signal at the RS, and each entry $\mathbf{T}_{l}$ is a unitary matrix. The codebook is constructed by grouping $2^{B}$ randomly generated unitary matrices. The BS and RS power constraints are given by $P_{t}=E\left[\left\|\mathbf{P}_{l} \mathbf{b}\right\|^{2}\right]=\operatorname{Tr}\left\{\mathbf{P}_{l} \mathbf{P}_{l}^{H}\right\}$ and $P_{r}=E\left[\left\|\mathbf{x}_{R}^{(l)}\right\|^{2}\right]=\beta^{2} \operatorname{Tr}\left\{\mathbf{T}_{l}\left(\mathbf{H}_{1} \mathbf{P}_{l} \mathbf{P}_{l}^{H} \mathbf{H}_{1}^{H}+\sigma_{1}^{2} \mathbf{I}\right) \mathbf{T}_{l}^{H}\right\}=$ $\beta^{2} \operatorname{Tr}\left\{\mathbf{H}_{1} \mathbf{P}_{l} \mathbf{P}_{l}^{H} \mathbf{H}_{1}^{H}+\sigma_{1}^{2} \mathbf{I}\right\}$, respectively, where $\operatorname{Tr}\{$.$\} de-$ notes the matrix trace operator. The scaling parameter $\beta$ is given by $\left.\beta=\sqrt{P_{r} / E\left[\left\|\mathbf{r}_{R}^{(l)}\right\|^{2}\right.}\right]$ which is computed at the RS.
For the second phase, we model the transmission from the RS to the $K \mathrm{MSs}$ as a MIMO broadcast channel, we stack all the users' received data and obtain the $K \times 1$ vector

$$
\mathbf{r}_{M}^{(l)}=\mathbf{H}_{2} \mathbf{x}_{R}^{(l)}+\mathbf{n}_{2}=\beta \mathbf{H}_{2} \mathbf{T}_{l} \mathbf{H}_{1} \mathbf{P}_{l} \mathbf{b}+\beta \mathbf{H}_{2} \mathbf{T}_{l} \mathbf{n}_{1}+\mathbf{n}_{2},
$$

where $\mathbf{H}_{2}$ is the $K \times N_{r}$ channel matrix between the RS and the MSs, its entries are i.i.d. zero mean complex Gaussian variables with unit variance, and $\mathbf{n}_{2}$ denotes the additive complex Gaussian noise, $E\left[\mathbf{n}_{2} \mathbf{n}_{2}^{H}\right]=\sigma_{2}^{2} \mathbf{I}$, where $\sigma_{2}^{2}$ denotes the second phase noise variance. Note that the matrix $\mathbf{H}_{2} \mathbf{T}_{l} \mathbf{H}_{1}$ is the $l$-th permutated equivalent channel matrix produced by the interleaving matrix $l$. The permutated channel matrices have different degrees of spatial orthogonality. For a given set of CSIs, we choose the interleaving matrix that provides the best spatial orthogonality for the proposed precoding scheme.

\section{Proposed SR-BAsed Robust Precoding STRUCTURE}

The CSI errors can be modeled as: $\mathbf{H}_{1}=\hat{\mathbf{H}}_{1}+\triangle \mathbf{H}_{1}$ and $\mathbf{H}_{2}=\hat{\mathbf{H}}_{2}+\triangle \mathbf{H}_{2}$, where $\hat{\mathbf{H}}_{1}$ and $\hat{\mathbf{H}}_{2}$ are the first phase and second phase estimated channel matrices, respectively, and $\triangle \mathbf{H}_{1}$ and $\triangle \mathbf{H}_{2}$ are i.i.d. complex estimation error matrices. The error matrices are assumed to be Gaussian distributed with zero mean and $E\left[\triangle \mathbf{H}_{1} \triangle \mathbf{H}_{1}^{H}\right]=\sigma_{h_{1}}^{2} N_{t} \mathbf{I}$ and $E\left[\triangle \mathbf{H}_{2} \triangle \mathbf{H}_{2}^{H}\right]=\sigma_{h_{2}}^{2} N_{r} \mathbf{I}$, where $\sigma_{h_{1}}^{2}$ and $\sigma_{h_{2}}^{2}$ denote the variance of each element in error matrices $\triangle \mathbf{H}_{1}$ and $\triangle \mathbf{H}_{2}$, respectively. In practice, the estimated CSIs at the BS can be obtained by employing the feedback channels [13], [14]

The proposed SR-based precoding scheme employs $2^{B}$ parallel branches of precoders which are designed based on the permutated equivalent channel matrices generated by different interleaving matrices in the codebook. The optimum precoder is selected from the $2^{B}$ candidates by a certain criterion based on a given set of CSIs. In the following, we introduce the proposed robust precoding design for branch $l$, in the presence of channel estimation errors. 
Let us consider the following mean squared error criterion

$$
\zeta=E\left[\left\|\mathbf{b}-\left(\mathbf{H}_{2} \mathbf{T}_{l} \mathbf{H}_{1} \mathbf{P}_{l} \mathbf{b}+\mathbf{H}_{2} \mathbf{T}_{l} \mathbf{n}_{1}+\mathbf{n}_{2}\right)\right\|^{2}\right] .
$$

Note that the expectation is taken over all distributions of $\mathbf{b}$, $\mathbf{n}_{1}, \mathbf{n}_{2}, \triangle \mathbf{H}_{1}$ and $\triangle \mathbf{H}_{2}$. By substituting $\mathbf{H}_{1}=\hat{\mathbf{H}}_{1}+\triangle \mathbf{H}_{1}$ and $\mathbf{H}_{2}=\hat{\mathbf{H}}_{2}+\triangle \mathbf{H}_{2}$ into (4) and taking the expectation, we have

$$
\begin{aligned}
\zeta= & \operatorname{Tr}\left\{E \left[\mathbf{I}-\mathbf{P}_{l}^{H}\left(\hat{\mathbf{H}}_{1}^{H} \mathbf{T}_{l}^{H} \hat{\mathbf{H}}_{2}^{H}+\triangle \mathbf{H}_{1}^{H} \mathbf{T}_{l}^{H} \hat{\mathbf{H}}_{2}^{H}\right.\right.\right. \\
& \left.+\hat{\mathbf{H}}_{1}^{H} \mathbf{T}_{l}^{H} \triangle \mathbf{H}_{2}^{H}+\triangle \mathbf{H}_{1}^{H} \mathbf{T}_{l}^{H} \triangle \mathbf{H}_{2}^{H}\right)-\left(\hat{\mathbf{H}}_{2} \mathbf{T}_{l} \hat{\mathbf{H}}_{1}\right. \\
& \left.+\hat{\mathbf{H}}_{2} \mathbf{T}_{l} \triangle \mathbf{H}_{1}+\triangle \mathbf{H}_{2} \mathbf{T}_{l} \hat{\mathbf{H}}_{1}+\triangle \mathbf{H}_{2} \mathbf{T}_{l} \triangle \mathbf{H}_{1}\right) \mathbf{P}_{l} \\
& +\left(\hat{\mathbf{H}}_{2} \mathbf{T}_{l} \hat{\mathbf{H}}_{1}+\hat{\mathbf{H}}_{2} \mathbf{T}_{l} \triangle \mathbf{H}_{1}+\triangle \mathbf{H}_{2} \mathbf{T}_{l} \hat{\mathbf{H}}_{1}\right. \\
& \left.+\triangle \mathbf{H}_{2} \mathbf{T}_{l} \triangle \mathbf{H}_{1}\right) \mathbf{P}_{l} \mathbf{P}_{l}^{H}\left(\hat{\mathbf{H}}_{1}^{H} \mathbf{T}_{l}^{H} \hat{\mathbf{H}}_{2}^{H}+\triangle \mathbf{H}_{1}^{H} \mathbf{T}_{l}^{H} \hat{\mathbf{H}}_{2}^{H}\right. \\
& \left.+\hat{\mathbf{H}}_{1}^{H} \mathbf{T}_{l}^{H} \triangle \mathbf{H}_{2}^{H}+\triangle \mathbf{H}_{1}^{H} \mathbf{T}_{l}^{H} \triangle \mathbf{H}_{2}^{H}\right) \\
& +\frac{\operatorname{Tr}\left\{\mathbf{P}_{l} \mathbf{P}_{l}^{H}\right\}}{P_{t}}\left(\sigma_{1}^{2} \hat{\mathbf{H}}_{2} \hat{\mathbf{H}}_{2}^{H}+\sigma_{1}^{2} \triangle \mathbf{H}_{2} \triangle \mathbf{H}_{2}^{H}\right) \\
& \left.\left.+\sigma_{2}^{2} \frac{\operatorname{Tr}\left\{\mathbf{P}_{l} \mathbf{P}_{l}^{H}\right\}}{P_{t}} \mathbf{I}\right]\right\}
\end{aligned}
$$

where we assume that $\operatorname{Tr}\left\{\mathbf{P}_{l} \mathbf{P}_{l}^{H}\right\}=P_{t}$ without loss of generality [15]. In the derivation we use the fact that $\mathbf{T}_{l}$ is a unitary matrix. By taking the gradient terms of (5) with respect to $\mathbf{P}_{l}^{*}$ and equating them to zero, we obtain

$$
\begin{aligned}
\nabla \zeta_{\mathbf{P}_{l}^{*}}= & -\left(\hat{\mathbf{H}}_{1}^{H} \mathbf{T}_{l}^{H} \hat{\mathbf{H}}_{2}^{H}\right)+\left(\hat{\mathbf{H}}_{1}^{H} \mathbf{T}_{l}^{H} \hat{\mathbf{H}}_{2}^{H} \hat{\mathbf{H}}_{2} \mathbf{T}_{l} \hat{\mathbf{H}}_{1}\right. \\
& +\sigma_{h_{1}}^{2} \operatorname{Tr}\left\{\hat{\mathbf{H}}_{2} \hat{\mathbf{H}}_{2}^{H}\right\} \mathbf{I}+\sigma_{h_{2}}^{2} K \hat{\mathbf{H}}_{1}^{H} \hat{\mathbf{H}}_{1} \\
& \left.+N_{r} K \sigma_{h_{1}}^{2} \sigma_{h_{2}}^{2} \mathbf{I}\right) \mathbf{P}_{l} \\
& +\frac{\operatorname{Tr}\left\{\sigma_{1}^{2} \hat{\mathbf{H}}_{2} \hat{\mathbf{H}}_{2}^{H}+\sigma_{1}^{2} \sigma_{h_{2}}^{2} N_{r} \mathbf{I}\right\}}{P_{t}} \mathbf{P}_{l}+\frac{K \sigma_{2}^{2}}{P_{t}} \mathbf{P}_{l}=\mathbf{0}
\end{aligned}
$$

where we used the statistical properties and the fact that $E\left[\triangle \mathbf{H}_{1}^{H} \mathbf{A} \triangle \mathbf{H}_{1}\right]=\sigma_{h}^{2} \operatorname{Tr}\{\mathbf{A}\} \mathbf{I}$ for any $N_{r} \times N_{r}$ matrix $\mathbf{A}$. Thus, we obtain the robust precoding expression as follows,

$$
\mathbf{P}_{l}=\boldsymbol{\Pi}^{-1} \hat{\mathbf{H}}_{1}^{H} \mathbf{T}_{l}^{H} \hat{\mathbf{H}}_{2}^{H},
$$

where $\boldsymbol{\Pi}=\hat{\mathbf{H}}_{1}^{H} \mathbf{T}_{l}^{H} \hat{\mathbf{H}}_{2}^{H} \hat{\mathbf{H}}_{2} \mathbf{T}_{l} \hat{\mathbf{H}}_{1}+\sigma_{h_{1}}^{2} \operatorname{Tr}\left\{\hat{\mathbf{H}}_{2} \hat{\mathbf{H}}_{2}^{H}\right\} \mathbf{I}+$ $\sigma_{h_{2}}^{2} K \hat{\mathbf{H}}_{1}^{H} \hat{\mathbf{H}}_{1}+N_{r} K \sigma_{h_{1}}^{2} \sigma_{h_{2}}^{2} \mathbf{I}+\frac{\sigma_{1}^{2} \operatorname{Tr}\left\{\hat{\mathbf{H}}_{2} \hat{\mathbf{H}}_{2}^{H}\right\}}{P_{t}} \mathbf{I}+\frac{K \sigma_{1}^{2} \sigma_{h_{2}}^{2} N_{r}}{P_{t}} \mathbf{I}+$ $\frac{K \sigma_{2}^{2}}{P_{t}} \mathbf{I}$. It is straightforward to see that for the $l$-th branch (7) is a global minimizer for the MMSE optimization problem since the Hessian matrix of the MSE objective function is positive definite. In order to satisfy the transmit power constraint $\operatorname{Tr}\left\{\mathbf{P}_{l} \mathbf{P}_{l}^{H}\right\}=P_{t}$, the proposed robust precoder for the $l$-th parallel branch is given by

$$
\mathbf{P}_{l}^{(o p t)}=\sqrt{\frac{P_{t}}{\operatorname{Tr}\left\{\mathbf{P}_{l} \mathbf{P}_{l}^{H}\right\}}} \mathbf{P}_{l} .
$$

Note that the $l$-th branch robust precoder is designed based on the permutated equivalent channel matrix $\mathbf{H}_{2} \mathbf{T}_{l} \mathbf{H}_{1}$ of the $l$-th interleaving matrix $\mathbf{T}_{l}$. The optimum precoder is chosen by a selection criterion to provide the best performance. In the next section, we will focus on the description of the proposed optimization selection criteria.

\section{OPTIMIZATION CRITERIA AND LOW-RATE FEEDFORWARD SCHEME}

In the proposed scheme, we assume that the BS and RS employ the same prestored codebook of interleaving matrices. In particular, the $\mathrm{BS}$ is equipped with a selection function and the estimated CSI for the first and second phases. In this section, the selection functions are introduced based on different optimization criteria. Then, we explain the details of the low-rate feedforward scheme and obtain an expression for the transmission efficiency.

\section{A. The Selection Criterion for Symbol Detection}

Firstly, we focus on the symbol detection problem to design the selection criterion. Note that the average symbol error rate performance is governed by the Euclidean distance between the true symbol value and its soft decision that is affected by the interference, noise and transmit symbols. By accumulating the values of the squared Euclidean distance in one transmission block, we have the following selection rule

$$
l_{\text {opt }}=\arg \min _{1 \leq l \leq 2^{B}}\left\{\sum_{j=1}^{K M}\left|\mathbf{s}_{j}(i)-\hat{\mathbf{u}}_{j}^{(l)}(i)\right|^{2}\right\},
$$

where the subscript $j$ denotes the $j$-th element of a vector, the quantity $\mathbf{s}(i)$ denotes the $i$-th transmission data block, which is given by $\mathbf{s}(i)=\left[\mathbf{b}^{T}(i), \ldots, \mathbf{b}^{T}(i+M-1)\right]^{T}, M$ is the block length, the $K \times 1$ vector $\mathbf{b}(i+m)$ denotes the $m$-th transmit vector of the $i$-th block, $m \in\{1, \ldots, M-1\}$, the soft decision vector $\hat{\mathbf{u}}^{(l)}(i)$ is given by $\hat{\mathbf{u}}^{(l)}(i)=\left[\hat{\mathbf{r}}^{T(l)}(i), \ldots, \hat{\mathbf{r}}^{T(l)}(i+M-\right.$ $1)]^{T}$, where $\hat{\mathbf{r}}^{(l)}(i+m)$ denotes the noiseless pre-estimated received vector based on the $l$-th interleaving matrix at the $\mathrm{BS}$, the structure is given by

$$
\hat{\mathbf{r}}^{(l)}(i+m)=\hat{\beta} \hat{\mathbf{H}}_{2} \mathbf{T}_{l} \hat{\mathbf{H}}_{1} \mathbf{P}_{l} \mathbf{b}(i+m),
$$

where $\hat{\beta}$ is the estimate for the RS scaling factor at the BS, by considering the channel estimate error we have the following expression

$$
\hat{\beta}=\sqrt{\frac{P_{r}}{\operatorname{Tr}\left\{\hat{\mathbf{H}}_{1} \mathbf{P}_{l} \mathbf{P}_{l}^{H} \hat{\mathbf{H}}_{1}^{H}\right\}+\operatorname{Tr}\left\{\sigma_{h_{1}}^{2} N_{r} \mathbf{P}_{l}^{H} \mathbf{P}_{l}\right\}+\sigma_{1}^{2} N_{r}}} .
$$

The optimum interleaving matrix is chosen by minimizing the summation of the squared Euclidean distance values in one transmission data block.

The final output of the $m$-th transmit vector of the $i$-th data block, i.e, $\hat{\mathbf{b}}_{f}(i+m)$ is given by

$$
\hat{\mathbf{b}}_{f}(i+m)=Q_{u}\left\{\mathbf{r}_{M}^{\left(l_{o p t}\right)}(i+m)\right\},
$$

where $Q_{u}\{$.$\} represents the quantization operation. \mathbf{r}_{M}^{\left(l_{\text {opt }}\right)}(i+$ $m)$ is the $K \times 1$ received vector for all the destination users. The optimization algorithm for symbol detection is implemented at the BS and is summarized in Table I.

\section{B. The Selection Criterion for Sum Rate Optimization}

The sum rate of the proposed relay system for the $l$-th interleaving matrix is computed as follows [2]

$$
R^{(l)}=\frac{1}{2} \sum_{k_{0}=1}^{K} \log _{2}\left(1+\gamma_{k_{0}}^{(l)}\right)
$$


TABLE I

Proposed ALGORITHM FOR S YMBOL DETECTION

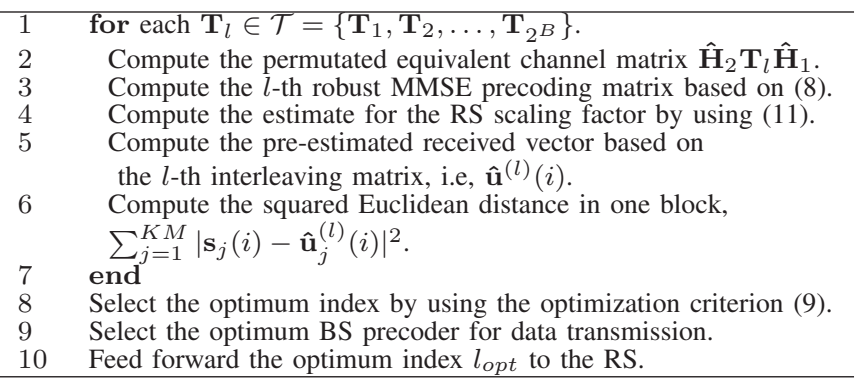

where $\gamma_{k_{0}}^{(l)}$ denotes the signal-to-interference-plus-noise ratio (SINR) of the $k_{0}$-th user for the $l$-th interleaving matrix, which is given by

$\gamma_{k_{0}}^{(l)}=\frac{\beta^{2} \overline{\mathbf{h}}_{k_{0}}^{(l)} \mathbf{p}_{k_{0}}^{(l)} \mathbf{p}_{k_{0}}^{(l) H} \overline{\mathbf{h}}_{k_{0}}^{(l) H}}{\sum_{k \neq k_{0}}^{K}\left(\beta^{2} \overline{\mathbf{h}}_{k_{0}}^{(l)} \mathbf{p}_{k}^{(l)} \mathbf{p}_{k}^{(l) H} \overline{\mathbf{h}}_{k_{0}}^{(l) H}\right)+\beta^{2} \sigma_{1}^{2} \mathbf{h}_{2, k_{0}}^{H} \mathbf{h}_{2, k_{0}}+\sigma_{2}^{2}}$,

where $k_{0} \in\{1, \ldots, K\}$, the $N_{r} \times 1$ vector $\mathbf{h}_{2, k_{0}}$ is the $k_{0^{-}}$ th column of the channel matrix $\mathbf{H}_{2}^{T}$, i.e. the second phase channel vector for user $k_{0}$, and the vector $\overline{\mathbf{h}}_{k_{0}}^{(l)}$ is given by $\overline{\mathbf{h}}_{k_{0}}^{(l)}=\mathbf{h}_{2, k_{0}}^{T} \mathbf{T}_{l} \mathbf{H}_{1}$.

By taking the imperfect CSI into account and using the statistical property of the channel estimation errors, we propose the following selection criterion at the BS for sum rate optimization

$$
l_{\text {opt }}=\arg \max _{1 \leq l \leq 2^{B}}\left\{\frac{1}{2} \sum_{k_{0}=1}^{K} \log _{2}\left(1+\hat{\gamma}_{k_{0}}^{(l)}\right)\right\},
$$

where the estimated SINR $\hat{\gamma}_{k_{0}}^{(l)}$ is given by

$$
\hat{\gamma}_{k_{0}}^{(l)}=\frac{\hat{\beta}^{2} \mathbf{p}_{k_{0}}^{(l) H} \boldsymbol{\Lambda} \mathbf{p}_{k_{0}}^{(l)}}{\sum_{k \neq k_{0}}^{K}\left(\hat{\beta}^{2} \mathbf{p}_{k}^{(l) H} \boldsymbol{\Lambda} \mathbf{p}_{k}^{(l)}\right)+\eta},
$$

where $\boldsymbol{\Lambda}=\hat{\mathbf{H}}_{1}^{H} \mathbf{T}_{l}^{H} \hat{\mathbf{h}}_{2, k_{0}}^{*} \hat{\mathbf{h}}_{2, k_{0}}^{T} \mathbf{T}_{l} \hat{\mathbf{H}}_{1}+\sigma_{h_{2}}^{2} \hat{\mathbf{H}}_{1}^{H} \hat{\mathbf{H}}_{1}+$ $\sigma_{h_{1}}^{2} \operatorname{Tr}\left\{\hat{\mathbf{h}}_{2, k_{0}}^{*} \hat{\mathbf{h}}_{2, k_{0}}^{T}\right\} \mathbf{I}+\sigma_{h_{1}}^{2} \sigma_{h_{2}}^{2} N_{r} \mathbf{I}$, and $\eta=\hat{\beta}^{2} \sigma_{1}^{2} \hat{\mathbf{h}}_{2, k_{0}}^{H} \hat{\mathbf{h}}_{2, k_{0}}+$ $\hat{\beta}^{2} \sigma_{1}^{2} N_{r} \sigma_{h_{2}}^{2}+\sigma_{2}^{2}$. Here, we have $\mathbf{h}_{2, k_{0}}=\hat{\mathbf{h}}_{2, k_{0}}+\triangle \mathbf{h}_{2, k_{0}}$, $\hat{\mathbf{h}}_{2, k_{0}}$ denotes the estimated channel vector for user $k_{0}$ in the second phase, and $\triangle \mathbf{h}_{2, k_{0}}$ is the channel error vector. The optimization algorithm for sum rate maximization which is implemented at the BS is summarized in Table II.

\section{Low-Rate Feedforward Block Structure}

In this work, the proposed limited feedforward scheme is based on sending the quantization bits of the interleaving matrix codebook. From the aforementioned discussion about the proposed scheme, we know that for every block prior to payload transmission, there is a preamble transmission in order to inform the RS of the optimum index of the relay interleaving matrix. The optimum index is chosen by the selection function at the BS based on estimated CSIs, and fed forward to the RS before the payload transmission. We insert the limited feedforward bits at the beginning of the corresponding transmission block. In the case of the selection criterion for symbol detection, the block of the multiantenna
TABLE II

\section{PROPOSED ALGORITHM FOR SUM RATE OPTIMIZATION}

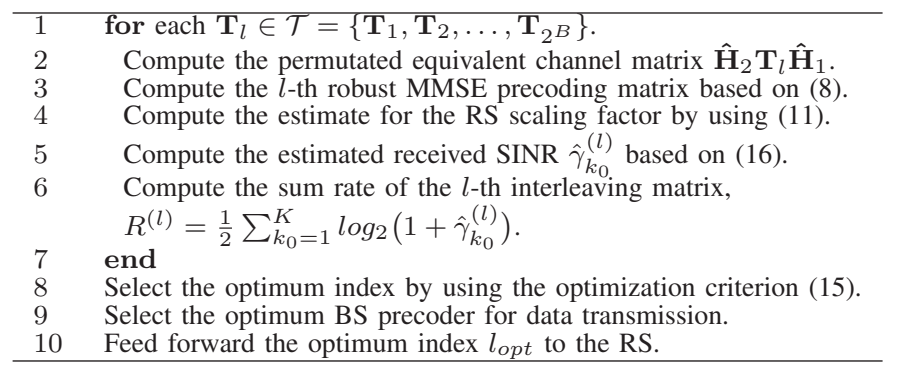

scheme comprises $M$ symbol periods each one consisting of $K$ spatial streams, and the feedforward rate of the optimum index is one per transmission block. Let us recall that $B$ bits can represent $2^{B}$ interleaving matrices and assume that $Q$-ary modulation is employed for the proposed SR-based scheme, thus for each block a number of $B$ feedforward bits need to be transmitted, while a number of $K M$ data symbols are transmitted. A number of $B$ signalling bits need to be sent for every $K M \log _{2}(Q)$ transmitted bits in the block. Therefore, the transmission efficiency is given by

$$
\varepsilon=\frac{K M \log _{2}(Q)}{K M \log _{2}(Q)+B} .
$$

In this work, for symbol detection we focus on the QPSK modulation and employ a data block of $M=8$ symbols in the simulation. For a configuration with $N_{t}=N_{r}=K=6$, by using $B=6$ feedforward bits we achieve the transmission efficiency of $94.12 \%$. In the case of the selection criterion for sum rate optimization, the index will be only updated when the channel changes. For a slow fading channel, the feedforward rate is very low, and the transmission efficiency is close to 1. In the following section, we will show the proposed SR-based scheme can improve the performance significantly.

\section{Simulations}

In this section, we evaluate the performance of the proposed SR-based robust precoding scheme and compare it with the existing precoding algorithms for multiuser MIMO relaying systems [3]-[7]. We assume that both the first phase MIMO channel and the second phase MIMO broadcast channel are quasi-static flat fading channels with Rayleigh distribution. 10000 channel realizations are employed for each simulation. The configuration of the system is $N_{t}=N_{r}=K=6$. We set $P_{t}=P_{r}=K$, and define the input $\mathrm{SNR}=P_{t} / \bar{\sigma}^{2}$, where $\bar{\sigma}=\sigma_{1}=\sigma_{2}$. Among the proposed techniques, we consider:

1) Proposed: the SR-based robust precoding algorithm.

2) $B$-bit: the limited feedforward schemes employ $B$ bits, namely $2^{B}$ is the codebook length.

3) Robust Identity: the proposed robust precoder (8) without codebook employing an identity matrix as the relay processing matrix.

4) Robust Relay MMSE: the MMSE-based robust MIMO RS precoding algorithm proposed in [3].

5) SVD-ZF: the SVD-based joint BS and RS ZF precoding algorithm proposed in [5].

6) SVD-RZF: the SVD-based robust joint BS and RS ZF precoding algorithm proposed in [6], [7]. 
Initially, we show the simulation results when the selection criterion is chosen for symbol detection. In the simulation, QPSK modulation is used. The channel estimate error variance is given by $\sigma_{h_{1}}^{2}=\sigma_{h_{2}}^{2}=0.003$. The channel varies per transmit block, each block contains $M=8$ symbols. The optimization algorithm in table I is utilized. Fig. 2 shows the average SER versus the SNR for comparing the proposed SR-based precoding scheme with the existing relay precoding schemes. The performance of the proposed SR-based robust MMSE precoding scheme is much better than the others. In particular, the proposed SR-based robust precoder with 6 bits can save over $3 \mathrm{~dB}$ in comparison with the robust relay MMSE precoder, at the average SER level of $2 \times 10^{-2}$. The SER performance of the SR-based precoding scheme with 6 bits under perfect CSI is given for reference.

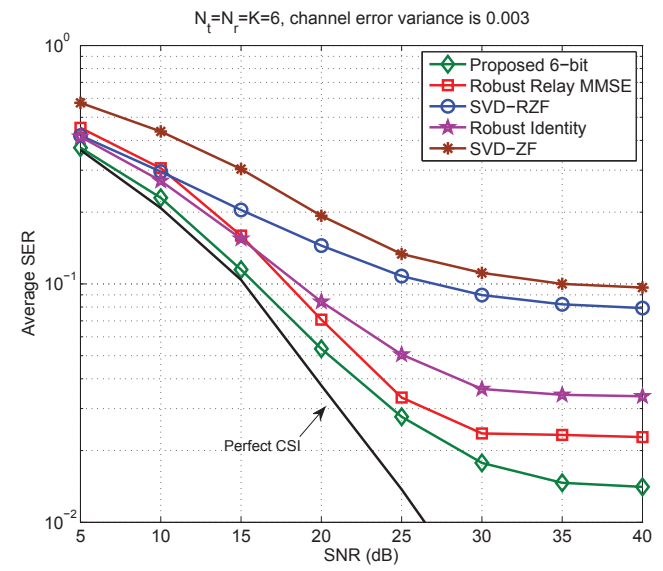

Fig. 2. Average SER performance versus SNR for the proposed SR-based robust precoding scheme and the existing relay precoding algorithms.

In the next experiment, we focus on the selection criterion for sum rate optimization. The optimization algorithm in table II is employed. The channel estimate error variance is given by $\sigma_{h_{1}}^{2}=\sigma_{h_{2}}^{2}=0.04$. Fig. 3 indicates the sum rate performance curves versus the SNR for the analyzed schemes. Firstly, we can find that the sum rate of the proposed algorithm increases as the number of feedforward bits increases. The results show that the best performance is achieved with the proposed SR-based robust precoding scheme with 12 feedforward bits, followed by the SVD-RZF precoding algorithm, the robust identity technique, the SVD-ZF precoding algorithm and the robust relay MMSE precoding algorithm. The sum rate performance of the SR-based precoding scheme with 10 bits under perfect CSI is given for reference. In particular, the proposed robust precoding scheme with 12 feedforward bits can save up to $5 \mathrm{~dB}$ in comparison with the SVD-RZF precoding algorithm, at the sum rate level of $6.5 \mathrm{bits} / \mathrm{Hz} / \mathrm{s}$.

\section{CONCLUSION}

In this paper, we have proposed a robust MMSE BS precoding strategy based on SR processing for multiuser MIMO relaying systems. We have also developed two types of selection functions, which were used for symbol detection and sum rate optimization, respectively. The transmission efficiency of the proposed block structure were investigated. The results have shown that the proposed SR-based scheme significantly outperforms the existing relay precoding algorithms, in the

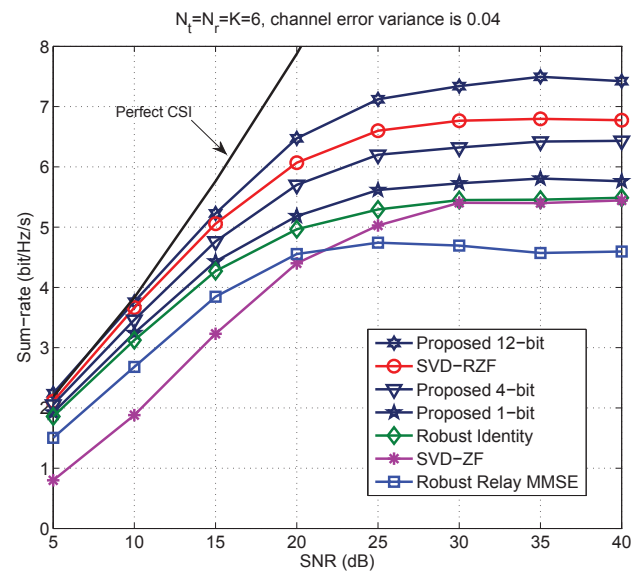

Fig. 3. Sum rate performance versus SNR for the proposed SR-based linear precoding scheme and the existing relay precoding algorithms.

presence of imperfect CSI. We remark that our proposed algorithms also can be extended to take into account systems with other precoding schemes.

\section{REFERENCES}

[1] R. Zhang, C. C. Chai, and Y. C. Liang, "Joint beamforming and power control for multiantenna relay broadcast channel with QoS constraints," in IEEE Trans. Signal Process., vol. 57, No. 2, pp. 726-737, Feb. 2009.

[2] C. Chae, T. Tang, R. W. Heath, Jr., and S. Cho, "MIMO relaying with linear processing for multiuser transmission in fixed relay networks," in IEEE Trans. Signal Process., vol. 56, no. 2, pp. 727-738, Feb. 2008.

[3] B. Zhang, Z. He, K. Niu and L. Zhang, "Robust linear beamforming for MIMO relay broadcast channel with limited feedback," in IEEE Signal Process. Lett., vol. 17, no. 2, Feb. 2010.

[4] P. Clarke ad R. C. de Lamare, "Transmit diversity and relay selection algorithms for multi-relay cooperative MIMO systems", IEEE Transactions on Vehicular Technology, vol. 61, no. 3, March 2012, pp. 1084 1098.

[5] W. Xu, X. Dong, and W.-S. Lu, "Joint optimization for source and relay precoding under multiuser MIMO downlink channels," in Proc. IEEE ICC 2010.

[6] W. Xu, X. Dong, and W.-S. Lu, "MIMO relaying broadcast channels with linear precoding and quantized channel state information feedback," in IEEE Trans. Signal Process., vol. 58, no. 10, Oct. 2010.

[7] Z. Wang, W. Chen, and J. Li, "Efficient beamforming for MIMO relaying broadcast channel with imperfect channel estimation," in IEEE Trans. Vehi. Tech., vol. 61, no. 1, Jan. 2012.

[8] F. Tseng and W. Wu, "Linear MMSE transceiver design in amplify-andforward MIMO relay systems," in IEEE Trans. Vehi. Tech., vol. 59, no. 2, pp. 754-765, Feb. 2010.

[9] F. Tseng, W. Wu, and J. Wu "Joint source/relay precoder design in nonregenerative cooperative systems using an MMSE criterion," in IEEE Trans. Wireless Commun., vol. 8, no. 10, pp. 4928-4933, Oct. 2009.

[10] Y. Cai, R. C. de Lamare and R. Fa, "Switched interleaving techniques with limited feedback for interference mitigation in DS-CDMA systems," IEEE Trans. Commun., vol.59, no. 7, pp. 1946-1956, Jul. 2011.

[11] Y. Cai, R. C. de Lamare and D. Le Ruyet, "Transmit processing techniques based on switched interleaving and limited feedback for interference mitigation in multiantenna MC-CDMA systems," IEEE Trans. Vehi. Tech., vol.60, no. 4, pp. 1559-1570, May 2011.

[12] J. N. Laneman and G. W. Wornell, "Cooperative diversity in wireless network: Efficient protocols and outage behaviour," in IEEE Trans. Inf. Theory, vol. 50, no. 12, pp. 3062-3080, Dec. 2004. 2007.

[13] D. J. Love, R. W. Heath, Jr., V. K. N. Lau, D. Gesbert, B. D. Rao and M. Andrews, "An overview of limited feedback in wireless communication systems," in IEEE Journal. Selected Areas In Commun., vol. 26, no. 8, pp. 1341-1365, Oct. 2008

[14] P. Ding, D. J. Love, and M. D. Zoltowski, "Multiple antenna broadcast channels with shape feedback and limited feedback," IEEE Trans. Sig. Proc., vol. 55, no. 4, Jul. 2007, pp. 3417-3428.

[15] A. D. Dabbagh and D. J. Love, "Multiple antenna MMSE based downlink precoding with quantized feedback or channel mismatch," in IEEE Trans Commu., vol. 56, no. 11, pp. 1859-1868, Nov. 2008. 